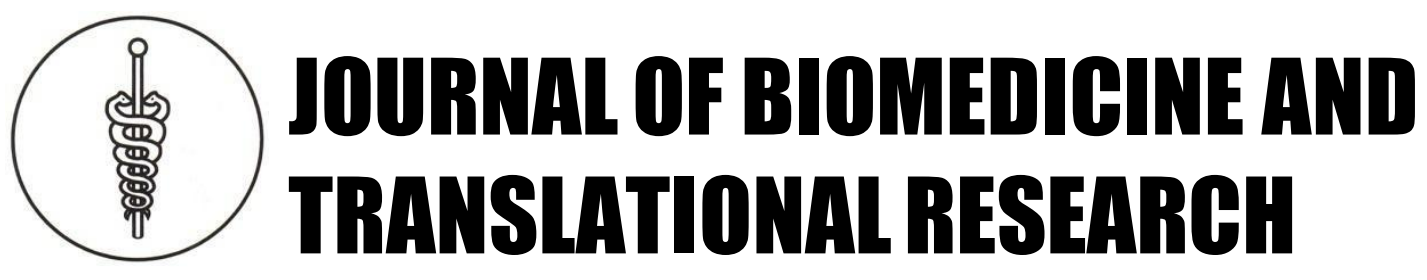

Copyright $@ 2017$ by Faculty of Medicine Diponegoro University and Indonesian Doctor Association, Central Java Region

\title{
Multidisciplinary Management of Disorders of Sex Development in Indonesia, A Prototype for Developing Country
}

\author{
Nurin Aisyiyah Listyasari ${ }^{1}$, Ardy Santosa ${ }^{2}$, Achmad Zulfa Juniarto ${ }^{3,4}$, Sultana MH Faradz ${ }^{3,4}$ \\ ${ }^{1}$ Graduate Student of Genetic Counseling, Master of Biomedical Science, Faculty of Medicine Diponegoro \\ University Semarang, Indonesia \\ ${ }^{2}$ Division Urology, Department of Surgery, Dr. Kariadi Hospital, Semarang, Indonesia \\ ${ }^{3}$ Division of Human Genetics Center for Biomedical Research (CEBIOR) Faculty of Medicine Diponegoro \\ University Semarang, Indonesia \\ ${ }^{4}$ Sexual Adjustment Team Dr. Kariadi Hospital / Faculty of Medicine Diponegoro University Semarang, \\ Indonesia
}

\section{Article info}

History :

Received : 15 May 2016

Accepted : 20 June 2017

Available : 23 June 2017

\begin{abstract}
Background : Disorder of sex development (DSD) patients require comprehensive management to improve quality of life. A standardized management protocol for patients in Indonesia is not yet available resulting in patients infrequently received a proper diagnosis and appropriate gender assignment.

Objectives : The purpose of the study was to report the management of DSD patients in Indonesia with regards to minimal diagnostic facilities and expertise in developing country.

Methodology : We analyzed DSD patients managed by Sexual Adjustment Multidisciplinary Team (SAT) through the record at Center for Biomedical Research Faculty of Medicine Diponegoro University, from May 2004 - December 2015 and bimonthly multidisciplinary SAT meeting. The team was consist of endocrinologist, andrologist, urologist, plastic surgeon, gynaecologist, anesthesiologist, medical geneticist, psychiatrist, psychologist, pathology, legal medicine, religion and social-medical staffs. Data were grouped by diagnosis that based on physical examination, family pedigree, karyotyping, hormonal assays and other examination such as ultrasonography, X-ray, and cytoscopy for selected cases.

Results : From the total of 617 cases we found 426 cases with 46,XY DSD $(69.04$ $\%), 117$ cases with 46,XX DSD (18.96\%), and 74 cases with sex chromosome DSD (12\%). Most of the patients in the group of 46,XY DSD were Unknown Male Undervirilization (60.09\%) followed by Congenital Adrenal Hyperplasia $(69.23 \%$ ) in 46, XX DSD group.

Conclusion : Comprehensive management for DSD Patients help patient in diagnosis, gender assignment and support patient to improve quality of life. This multidisciplinary of DSD team is the only team in Indonesia that can be used as a model for other center in Indonesia as well as other developing countries with minimal diagnostic facilities.
\end{abstract}

Keywords: Disorder of Sex Development (DSD); Sexual Adjustment Team; Chromosome. 


\section{INTRODUCTION}

Disorder of Sex Development (DSD) is defined by congenital conditions in which development of chromosomal, gonadal, or anatomical sex is atypical. ${ }^{1}$ This broad category is divided into three primary roots based on karyotype namely 46,XY DSD; 46,XY DSD and Sex Chromosome DSD. Secondary root is depicted based on hormone assessment and imaging, while the tertiary root is obtained based on molecular and/or histopathology examination.

In Indonesia, it is very rare to have a complete diagnosis procedure where molecular evaluation is needed for DSD confirmation. Molecular analysis as part of health care services is limited to research center of the universities in big cities. Early diagnosis and integrated treatment are important for DSD patients, in order to anticipate the proper treatment and gender assignment within medical, psychological and social aspect through the gender team.

A number of studies from different countries and cultural differences showed that while many patients managed sequentially, gender counseling, as well as sexual counseling, should be the part of multidisciplinary service available to patients with DSD to avoid gender dysphoria. ${ }^{2,3}$ The aim of this study is to review the management of DSD patients in Indonesia as a developing country related to providing appropriate gender assignment and to improving patients quality of life.

\section{METHODOLOGY}

The formation of Sexual Adjustment Multidisciplinary Team (SAT) since 1989, is the only team available in Indonesia with the members from Dr. Kariadi Hospital and Faculty of Medicine Diponegoro University (FMDU), which consists of specialists from various departments : urology, plastic surgery, gynecology, endocrinology, andrology, anesthesiology, genetics, psychiatry, pathology, psychology, legal medicine, religion and socialmedical staffs. The purpose of the Team is to provide the following comprehensive care for families and individuals with DSD.

The activities performed by SAT are bimonthly meetings, special multidisciplinary meetings for case discussion of about 5-10 cases, clinical and laboratory examination, patient's home visits, scientific meetings and overseas collaboration for research project.

We analyzed DSD patients managed by SAT through the record and bimonthly multidisciplinary SAT meeting at Center for Biomedical Research Faculty of Medicine Diponegoro University, from May 2004 - December 2015. Data were grouped by diagnosis based on new DSD classification by Hughes. 4

\footnotetext{
* Corresponding author:

Prof. Sultana MH Faradz, MD, PhD

Phone / Fax : 62-24-8454714

Email : sultanafaradz@gmail.com
}

Diagnosis was established by physical examination, family pedigree, karyotyping, hormonal assays, and other examination such as ultrasonography, X-ray, and Cytoscopy for selected cases. The patients were referred by clinicians from the department of urology, pediatrics, internal medicine, obstetrics, and member of sexual adjustment team. The reason for referral was the presence of ambiguous genitalia or any anatomical abnormality of external or internal genitalia. However, a few may present with delayed puberty or primary amenorrhea. Each patients with genital disorder is referred to the SAT member, with the goal of establishing the chromosomal sex, the gonadal sex, and the phenotypic sex (appearance of the external and internal genitalia) to determine the underlying diagnosis.

Approval from the medical ethics committee of FMDU and informed consent from all participants, or parents / guardians prior to their participation in this study were obtained.

\section{RESULT}

A total of 617 DSD patients were evaluated by SAT member. The age of patients were ranging from 2 days to 44 years old. Patients referred after the age of one year and adults were dominant among our patients (see table 1). Patients data were categorized according to their diagnosis; 426 cases (69.04\%) with 46,XY DSD, 117 cases (18.96\%) with 46,XX DSD and 74 cases $(12 \%)$ with sex chromosome DSD (Figure 1). Most of the patients in the group of 46,XY DSD were 256 cases $(60.09 \%)$ with Unknown Male Undervirilization (UMU) (Figure 2) and in the group 46,XX DSD were 81 cases $(69.23 \%)$ with Congenital Adrenal Hyperplasia. (Figure 3).

The remaining cases of 46,XY DSD group were 140 cases $(32.86 \%)$ with Androgen Action Disorder (AAD) and 30 cases (7.04\%) with Gonadal Dysgenesis, while 46, XX DSD groups were Androgen Excess Disorders 3 cases (2.56\%), Defect of Mullerian Development with 19 cases (16,24\%), 3 cases $(2.56 \%)$ of Androgen Excess, 3 cases $(2.56 \%)$ of 46, XX DSD Gonadal Dysgenesis And 74 cases with Sex Chromosome DSD.

\section{DISCUSSION}

The SAT in our center is the only multidisciplinary team for managing DSD cases so far. The medical management approach to DSD is based on the belief that DSD is pathological and devalues the experience of affected individuals. The management of DSD must always be undertaken on a case-by-case basis with the goal of maximizing longterm physical and psychological outcomes for individuals with DSD. ${ }^{5}$ Patient came from different region of Indonesia with different tribe, culture and religion, therefore management DSD in Indonesia should be carried out individually.

In Indonesia, as well as in South Asian countries, genetic counselor as health care provider is not very common, therefore clinical physicians would have to 
deal with patients and family members by their own way quite often. ${ }^{6}$ The majority cases of 46, XX DSD was Congenital Adrenal Hyperplasia (CAH) (81 cases) which required immediate comprehensive treatment because its nature of the crisis often leads to a life-threatening situation. Unfortunately, most of the medication for $\mathrm{CAH}$ is not available in Indonesia. Donation based on research was obtained from our collaborator such Erasmus University Medical Center Rotterdam, The Netherlands.

DSD is a broad term that describes a large variety of phenotype with disturbed development of internal and external genitalia, which is the result of various events occurring during sex differentiation. There are potentially novel genes and alternative pathways may play that lead to gonadal formation and differentiation, it is not always possible to identify the causes of the disorders. ${ }^{7-9}$ Consequently, the scope of molecular genetic analysis has markedly expanded. In Indonesia laboratory with molecular diagnosis facilities is very rare and advanced molecular testing are only available in big cities. Although clinical and psychological management have already been done, many undiagnosed DSD should be later analyzed using advanced molecular techniques. ${ }^{10}$

The wide spectrum of the clinical presentation of DSD is another aspect, and the interval of detection from the antenatal period into childhood or adolescence is also a factor to consider. ${ }^{11,12}$ Financial resources, health insurance issues, social, and cultural variables can also influence the attitudes of the parents for gender preference of the newborn or child with DSD. Because of National Health Insurance (JKN) cannot cover for diagnostic testing and treatment of DSD, the funds sometimes came from fund rising. This is become big issues for patients and family. They should consider whether they have sufficient funds to undergo therapy. The distances to a referral hospital is the second issue that must be faced by patients and their families. The long distance means they have to leave their families at home and they can not go to work while going for medical consultation and treatment. They must consider not only the decision from him/ herself, but also must consider the decision of the whole family.

Many patients come in late condition with physical and psychological complex dilemma. In many of underprivileged Asian countries, a child born with ambiguous genitalia grow up while bearing the congenital anatomic sex features, which remain untreated in the form of surgical treatment until adolescences or adulthood. ${ }^{2,3,13}$

Psychological evaluation was done by our group in the previous study to identify the concerns of the parents of a newborn with ambiguous genitalia and provide support for the accompanying uncertainty. ${ }^{14}$ This evaluation includes an assessment of parents' expectations regarding the gender of their baby, their understanding of what they have been told since the baby's birth, and their preferences, particularly within the context of their cultural and religious background, as well as a general assessment of coping strategies. ${ }^{14}$
Adult untreated patients either they already changed their gender or still undecided gender were suffered from stigmatization and gender dysphoria and need thorough psychological assessment. ${ }^{15-17}$ Culture is an important part of the context in which decisions are made on sex assignment. Attitudes concerning gender and sexuality, including the acceptance of intersexuality, may differ between various cultures. ${ }^{2,6}$ In our study, cultural differences as well as religion in dealing with DSD individuals not only influence the patient's own psychosexual development but also medical decisions regarding sex assignment and consecutive management.

The majority of well-established diagnosis were Androgen Insensitivity Syndrome and Congenital Adrenal Hyperplasia. These diagnoses already been proven by $A R$ gene and $C Y P 21$ mutation in 46, XY and 46, XX DSD respectively. ${ }^{18}$

In Indonesia, diagnosis and management of DSD patients have not received attention they deserve, since infectious disease is still a priority as the major cause of death. The first multidisciplinary approach on DSD management conducted in Indonesia is held in our center. Once we provided a multidisciplinary service and publicized to the community, a lot of patients came for consultation. Lacks of expertise, laboratory equipment and financial burden, as well as awareness of medical personnel and community were slow down its developments. The genital abnormality seems culturally taboo for some ethnic group to be disclosed to the family and medical personnel. The accountable general guideline of DSD management should be set and standardized nationally; hence it will provide adjustable implementation for the various conditions of patients and their families. Therefore, DSD management should be employed differently along with the diversity of cultural and demographic background in the societies.

Understanding on this issue, including an extensive research of patients and identify the structure of each ethnic and cultural background is necessary for general medical community in Indonesia in the management of patients with DSD complex. This report is expected to contribute to DSD patients, suggestions, advice or recommendations on what is needed in the diagnosis and management of DSD which will ultimately improve the quality of medical care and quality of life of DSD patients in Indonesia. Recognition, support, caring and providing the medication from the government should be soon become reality.

\section{CONCLUSION}

Comprehensive management for DSD Patients help patient in diagnosis, gender assignment and support patient to improve quality of life. To the best of our knowledge, this multidisciplinary of DSD team is the only team in Indonesia that can be used as a model for other center in Indonesia as well as other developing countries with minimal diagnostic facilities. 


\section{ACKNOWLEDGEMENT}

We thank Prof Stenvert L.S Drop who provided insight and expertise that greatly assisted the research, Sexual Adjustment Team Dr. Kariadi Hospital / Faculty of Medicine Diponegoro University and all patients who agreed to participate in this study.

\section{REFERENCES}

1. Hughes IA, Houk C, Ahmed SF, Lee PA, and LWPES1/ESPE2 Consensus group. Consensus statement on management of intersex. Archdischild. 2006;91:554-63.

2. Warne GL, Raza J. Disorders of sex development (DSDs), their presentation and management in different cultures. Rev Endocr Metab Disord. 2008;9(3):227-36.

3. Warne GL. Long-term outcome of disorders of sex development. Sex Dev. 2008;2(4-5):268-77.

4. Hughes IA, Houk C, Ahmed SF, Lee PA. Consensus statement on management of intersex disorders. $J$ Pediatr Urol. 2006;2(3):148-62.

5. Lathrop B, Cheney TB. Ethical perspectives on the management of disorders of sex development in children. Medicolegal Bioeth. 2015;5:27-34.

6. Zayts O, Sarangi S, Thong MK, Chung BHY, Lo IFM, Kan ASY, et al. Genetic counseling/consultation in South-East Asia: A report from the workshop at the 10th Asia pacific conference on human genetics. $J$ Genet Couns. 2013;22(6):917-24.

7. Piprek RP. Molecular and cellular machinery of gonadal differentiation in mammals. Int $J$ Dev Biol. 2010;54(5):779-86.

8. Kousta E, Papathanasiou A, Skordis N. Sex determination and disorders of sex development according to the revised nomenclature and classification in 46,XX individuals. Hormones. 2010;9(3):218-31.

9. Eggers S, Sinclair A. Mammalian sex determinationinsights from humans and mice. Chromosom Res. 012;20(1):215-38.

10. Faradz SM. Genetic analysis for the diagnosis of disorders of sexual development in Indonesia. J Biomed Transl Res. 2016;2:22-4.

11. Özbey H, Etker S. Disorders of sexual development in a cultural context. Arab J Urol. 2013;11(1):33-9.

12. Ediati A, Maharani N, Utari A. Sociocultural aspects of disorders of sex development. Embryo Today Rev. 2016;108(12):380-3.

13. Lee PA, Nordenström A, Houk CP, Ahmed SF, Auchus $\mathrm{R}$, Baratz A, et al. Global disorders of sex development update since 2006: Perceptions, approach and care. Horm Res Paediatr. 2016;85(3):158-80.

14. Ediati A, Faradz SMH, Juniarto AZ, van der Ende J, Drop SLS, Dessens AB. Emotional and behavioral problems in late-identified Indonesian patients with disorders of sex development. J Psychosom Res.
2015;79(1):76-84.

15. Ediati A, Juniarto AZ, Birnie E, Drop SLS, Faradz $\mathrm{SMH}$, Dessens AB. Gender development in Indonesian children, adolescents, and adults with disorders of sex development. Arch Sex Behav. 2015;44(5):1339-61.

16. Ediati A, Juniarto A, Birnie E, Okkerse J, de la Croix A, Wisniewski A, et al. Gender change and stigmatization in late-treated Indonesian children, adolescent, and adult patients with DSD. Int $J$ Pediatr Endocrinol. 2015;2015(Suppl 1):O56.

17. Ediati A, Juniarto AZ, Birnie E, Drop SLS, Faradz $\mathrm{SMH}$, Dessens AB. Body image and sexuality in indonesian adults with a disorder of sex development (DSD). J Sex Res. 2015;52(1):15-29.

18. Juniarto Z, van der Zwan YG, Santosa A, Ariani MD, Eggers S, Hersmus R, et al. Hormonal evaluation in relation to phenotype and genotype in 286 patients with a disorder of sex development from Indonesia. Clin Endocrinol (Oxf). 2016;0:1-11. 
Table 1. Age distribution of DSD patients at the time of referral

More than half of patients came to our clinic at later age (more than 1 years old).

\begin{tabular}{ccc}
\hline Age of referral & \multicolumn{2}{c}{ DSD Patients } \\
\hline$<6$ months old & Frequency $(\mathbf{n})$ & Percentage $(\boldsymbol{\%})$ \\
\hline months old $-<1$ year old & 74 & 11.99 \\
1 year old $-<5$ year old & 33 & 5.35 \\
5 year old $-<$ 10 year old & 169 & 27.39 \\
10 year old $-<15$ year old & 115 & 18.64 \\
$>$ = 15 year old & 107 & 17.34 \\
Total & 119 & 19.29 \\
\end{tabular}

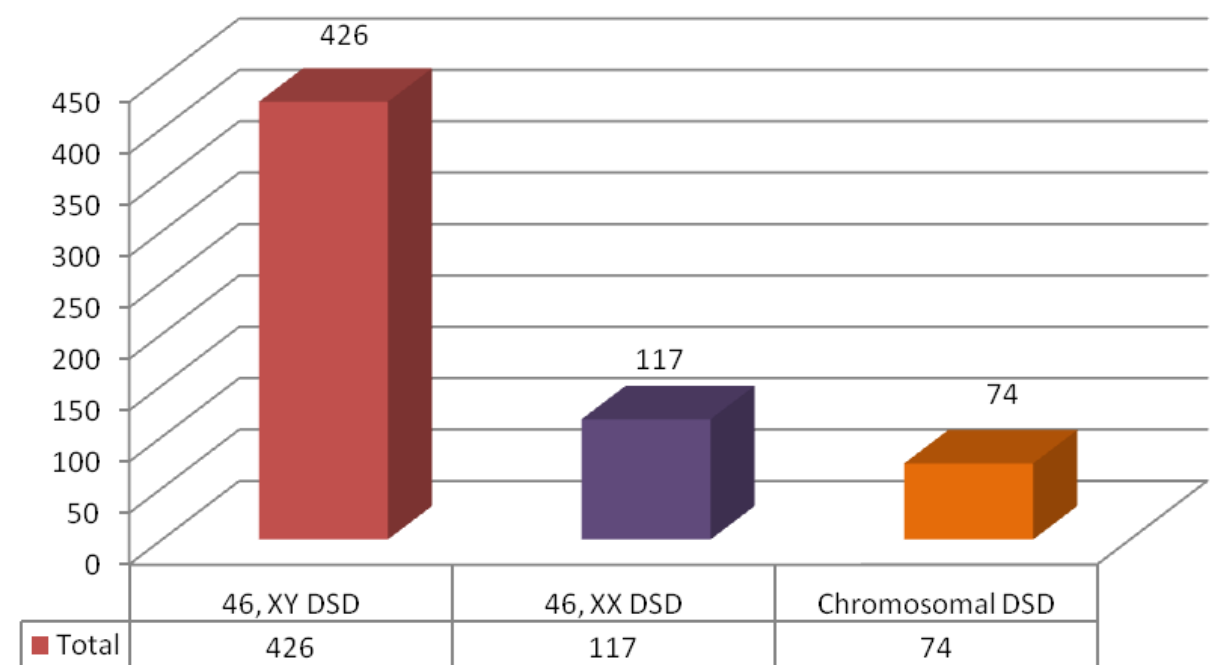

Figure 1. DSD Clasification

Six hundred and seventeen patients with various form of DSD were evaluated in our center. All the patients were categorized based on chromosome analysis result. There are 426 cases $(69,04 \%)$ with 46, XY DSD, 117 cases $(18,96 \%)$ with 46, XX DSD and 74 cases $(12 \%)$ with sex chromosome DSD.

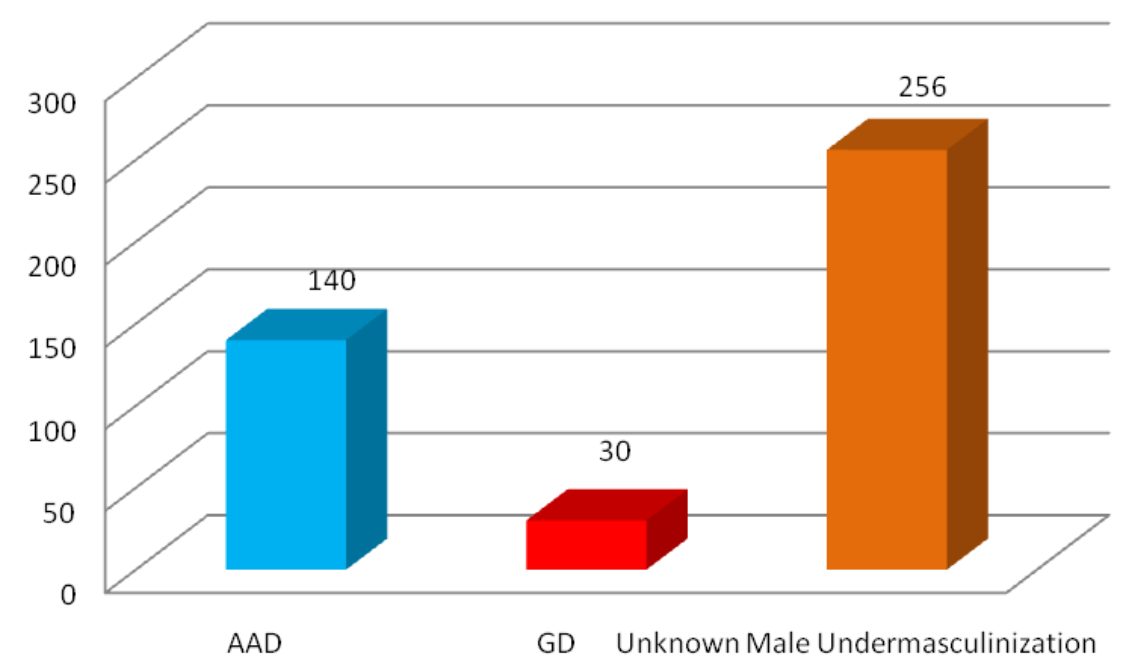

Figure 2. Distribution of 46,XY DSD patients

Most patients were UMU (60, 09\%) followed by Androgen Action Disorders (32.86\%) and Gonadal Dysgenesis $(7.04 \%)$ 


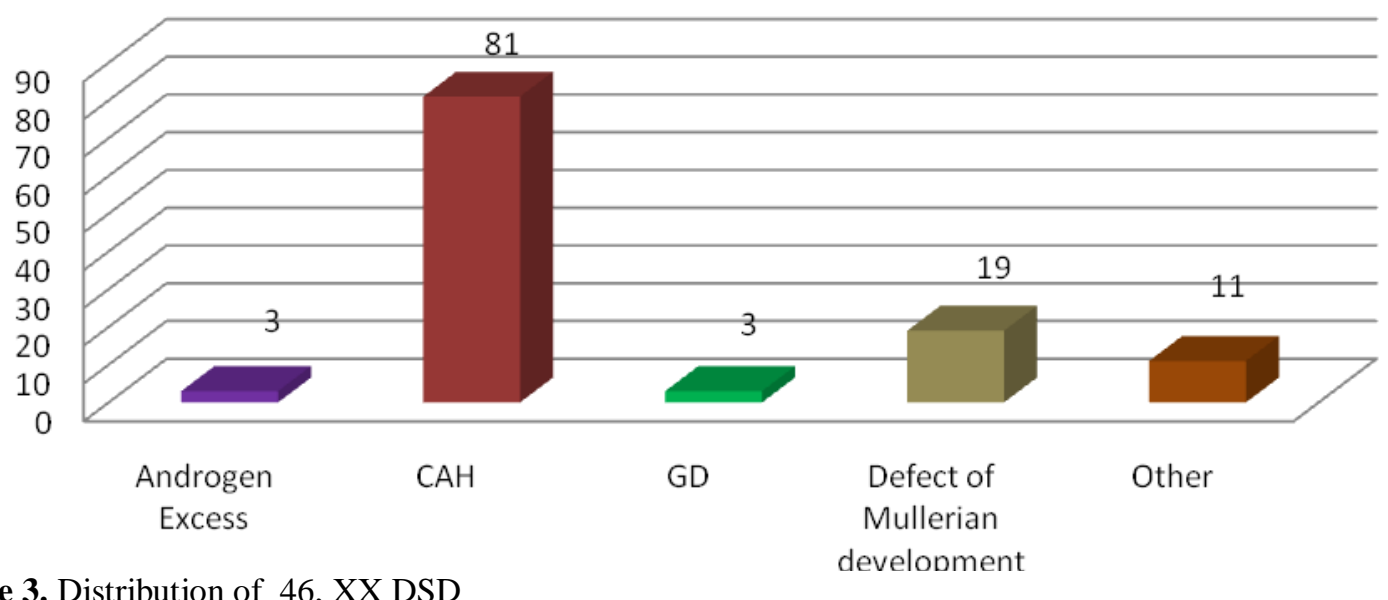

Figure 3. Distribution of 46, XX DSD

Most patients were Congenital Adrenal Hyperplasia (69.23\%)followed by Defect of Mullerian Development (16.24\%); Androgen Excess (2.56\%) and Gonadal Dysgenesis (2.56\%). 